\title{
Changes in structural and chemical composition of insoluble dietary fibers bound phenolic complexes from grape pomace by alkaline hydrolysis treatment
}

\author{
Guihun JIANG ${ }^{1 *}\left(\mathbb{D}\right.$, Zhaogen WU ${ }^{1 *}$, Karna RAMACHANDRA², Chen ZHAO ${ }^{1 *}$, Kashif AMEER ${ }^{3 *}$
}

\begin{abstract}
This study was aimed at explanation of evolution process of bound phenolics from IDF through structural modifications after alkaline hydrolysis along with free phenolics and functional characteristics. SEM results depicted that IDF with bound phenolics exhibited denser, more compact and tubular shape whereas, the surface features of alkali-hydrolyzed residue exhibited clear fragmentation, wrinkling and porosity on outside surfaces. Microstructure changes due to alkaline hydrolysis caused disintegration of linkages among hemicellulose and cellulose microfibers. As evident from the FTIR spectra, both IDF samples (with phenolic) and alkali-soluble residue (IDF without phenolics) exhibited resemblance in FTIR spectral features and characteristics bonds. Overall, the peak positions of IDF samples and alkali-hydrolyzed residue did not show any significant change, which was indicative of the fact that no major alterations were occurred in crystalline structures of IDF due to alkali-hydrolysis treatment. IDF-bound phenolic complexes showed the highest TPC, ABTS and FRAP than SDF. In IDF alkaline extracts, total 6 phenolic compounds were detected through HPLC. The identified phenolic compounds were as; salicylic acid, chlorogenic acid, syringic acid, epigallocatechin, $p$-coumaric acid and ferulic acid. These results demonstrated that higher antioxidant activity of dietary fiber would be related to bound phenolics.
\end{abstract}

Keywords: grape pomace; Vitis vinifera; insoluble dietary fiber; bound phenolics; alkaline hydrolysis.

Practical Application: Insoluble dietary fibers bound phenolic complexes can be exploited in formulation of nutraceuticals and functional food development.

\section{Introduction}

Non-digestible food ingredients exhibits significance as a broad category and dietary fiber (DF) is classified as one of them. Decreasing tendencies have been reported regarding risk factors and incidence of metabolic syndrome and cardiovascular diseases. As it is also ascertained previously that DF plays significant role, however, phenolics have also been reported to play pivotal role in prevention of aforementioned ailments (Xu et al., 2020). Phenolics are categorized as secondary metabolites derived from plant sources and they are vital to maintain human wellness and health (Liu et al., 2019a). Broadly, phenolics are classified in two types; bounds phenolic and free phenolics. In case of bounds phenolics, they are found in bounded form through covalently linked to plant matrix and aqueous solutions and binary solutions of organic extractants cannot extract bounds phenolics from plant matrices (Li et al., 2019). There is a need to be considered both free and bound phenolics in commutative manner to avoid the under-presenting of total phenolic contents (TPC) in reality. Hence, when TPC are measured then only free phenolics are taken into account as established in previously published reports (Xu et al., 2020).

During last decades, health benefits of both phenolics and DF have been studied in independent manner. In fact, DF is a rich source of phenolics (Guo \& Beta, 2013). In the large intensity, the phenolics found in bound form with DF have been reported to exert beneficial effects on overall human wellness owing to creation of antioxidant-rich environment. In a recent report by Maurer et al. (2019), it was implied that DF-bound phenolics may lead to restoration of cecal metabolism and may cause production of beneficial metabolites for reduction of activation of unfolded protein response. This was also indicative of synergistic effect of DF in combination with phenolics.

DF has been classified in two broad categories named insoluble dietary fiber (IDF) and soluble dietary fiber (SDF). Furthermore, the type and content of phenolics which combine to IDF and SDF may vary (Guo \& Beta, 2013). It was evident from one of the published reports that phenolics combined with IDF exhibited stronger antioxidant capacity as compared to that was found in SDF-phenolic combination. Hence, this implied that type and content of phenolics in IDF and SDF may affect the respective functions (Xu et al., 2020).

Grape is a seasonal fruit in various regions of the world including South East Asia, Europe and South Asia. Grape pomace has been reported to exhibit potent antioxidant potential owing 
to presence of rutin, quercetin, (-)-epigallocatechin (EGC) etc. It was also previously reported grape pomace contains rich quantities of bound phenolics (gallic acid, protocatechuic acid, salicylic acid, chlorogenic acid, syringic acid, epigallocatechin, catechin, $p$-coumaric acid, ferulic acid) (Monteiro et al., 2021). Published literature showed that antioxidant activity, structural properties and composition of grape pomace from various varieties may subject to variations (Drosou et al., 2015). It was assumed that removal of bound phenolics led to significant decline in antioxidant activities. However, the detailed evolution process has not been taken into rigorous investigation until now. Therefore, comparison of structural modifications of IDF-bounds phenolics prior to and after exposure to alkaline hydrolysis treatment might be help to ascertain the contribution of phenolics in total antioxidant capacity of DF (Monteiro et al., 2021).

Hence, the novelty of this study was aimed at explanation of evolution process of bound phenolics from IDF through structural modifications after alkaline hydrolysis along with free phenolics. In order to understand better the underpinning contribution of bounds phenolics originated from DF, the current study was focused on exploration of the structural alterations of IDF-bound phenolics prior to and after subjecting to alkaline hydrolysis (alkali-hydrolyzed residue) and functional characteristics by scanning electron microscopy (SEM), X-ray diffraction (XRD), and FTIR spectroscopy. Moreover, the IDF and SDF fractions were compared in terms of composition, TPC and cell wall components (cellulose, hemicellulose and lignin), antioxidant activities (ABTS and FRAP) of SDF and IDF-bound phenolics from grape pomace.

\section{Materials and methods}

\subsection{Materials}

Grape was procured from a local supermarket in Jillin city (longitude $125^{\circ} 40^{\prime} \sim 127^{\circ} 56^{\prime}$, latitude $42^{\circ} 31^{\prime} \sim 44^{\circ} 40^{\prime}$ ), China.

\subsection{Sample preparation}

The wet grape pomace after juice production were dried in a convection drying equipment at $55^{\circ} \mathrm{C}$, and continued till the moisture content reached less than $10 \%$. The dried grape pomace were then grounded to a powder and sifted through a 40-mesh sieve.

\subsection{Extraction of SDF}

The extraction of grape pomace SDF method was followed by Mañas \& Saura-Calixto (1993) with slight modification. Twenty milliliters of acetic acid-sodium acetate buffer were added to $10 \mathrm{~g}$ dried grape pomace. The sample was placed in a water bath at $100{ }^{\circ} \mathrm{C}$ for $1 \mathrm{~h}$, and then cooled to room temperature. Subsequently, $0.03 \%(\mathrm{w} / \mathrm{v})$ cellulase $(\mathrm{pH} 4.9)$ was added and allowed to react at $50{ }^{\circ} \mathrm{C}$ for $1.5 \mathrm{~h}$. The mixture was heated to $85^{\circ} \mathrm{C}$ for $10 \mathrm{~min}$. Papain $(0.06 \% \mathrm{w} / \mathrm{v})$ was added to the mixture in the water bath and allowed to react at $60^{\circ} \mathrm{C}$ for $30 \mathrm{~min}$. The mixture was cooled to room temperature, and four volumes of 95\% (v/v) ethanol were added to precipitate the SDF overnight.

\subsection{Extraction of IDF}

The extraction of grape pomace IDF as followed method described by Jiang et al. (2020). Ginseng residue was treated with heat-stable $\alpha$-amylase, protease and amyloglucosidase for the elimination of starches and proteins. Precipitate was dried at $50{ }^{\circ} \mathrm{C}$ for $24 \mathrm{~h}$ to derive IDF.

\subsection{Bound phenolic preparation by alkaline hydrolysis treatment}

The IDF and SDF were weighed, and $4 \mathrm{~mol} / \mathrm{L}$ of $\mathrm{NaOH}$ solution was added at the ratio of 1:20 for $90 \mathrm{~min}$ (Irakli et al., 2012). The sample was centrifuged at $8524 \times \mathrm{g}$ for $8 \mathrm{~min}$, and the supernatant was preserved. The residue was cleaned twice with distilled water. After drying, IDF alkaline hydrolysis residue (or SDF alkaline hydrolysis residue) without bound phenolics was obtained and weighed. The supernatant was used to determine the total phenolic content (TPC) and total phenolic content (TFC), and antioxidant activities.

\subsection{TPC}

The TPC of bound phenolic content in IDF and SDF was determined using the Folin-Ciocalteau reagent method according to methodology of Eghdami \& Sadeghi (2010). The measurements of TPC were expressed as $\mathrm{mg}$ gallic acid equivalent (mg GAE/100 g dry weight (DW).

\subsection{Antioxidant activity}

The ABTS and FRAP assays (antioxidant activities) were performed based to the methods described by Thaipong et al. (2006). Absorbance values of ABTS and FRAP assays were determined using a spectrophotometer at 734 and $593 \mathrm{~nm}$, respectively. The results were expressed as micromole Trolox equivalents ( $\mu \mathrm{mol} \mathrm{TE} / \mathrm{g}, \mathrm{DW})$. The standard curve made using different concentrations ( 25 and $800 \mathrm{mM}$ ) Trolox.

\subsection{Phenolic compounds}

Phenolic compounds from the bound phenolic content in IDF and SDF were determined using an Agilent 1260 Infinity chromatograph (Agilent Technologies, Waldbronm, Germany) equipped with a VWD detector. An HPLC C-18 column $(150 \times 4.6 \mathrm{~mm})$ was used at a flow rate of $1.0 \mathrm{~mL} / \mathrm{min}$ at $40^{\circ} \mathrm{C}$. Two phases were utilized to perform gradient elution using phase $A$ acetonitrile and phase B (glacial acetic acid:water, 1:99) under following gradient: $5 \% \mathrm{~A}(0-18 \mathrm{~min}) ; 5$ to $10 \% \mathrm{~A}(18-20 \mathrm{~min})$; 10 to $15 \% \mathrm{~A}(20-30 \mathrm{~min}) ; 15 \% \mathrm{~A}(30-32 \mathrm{~min}) ; 15$ to $28 \% \mathrm{~A}$ (32-55 min); 28 to $30 \%$ A (55-59 min); 30 to $45 \%$ A (59-60 min); 45 to $55 \% \mathrm{~A}(60-80 \mathrm{~min})$ and 5 to $55 \% \mathrm{~A}(80-81 \mathrm{~min}) .0 .45-\mu \mathrm{m}$ filter was utilized for sample filtration. Then, $10 \mu \mathrm{L}$ of samples and standard solutions were injected into HPLC system and chromatographic measurements for phenolic compounds were recorded at $280 \mathrm{~nm}$ detection wavelength. The phenolic compounds content was quantified by comparing retention times of GSI-enriched bread samples with those of the standard solution and results were expressed as $\mu \mathrm{g} / \mathrm{g}$ DW. 


\subsection{Cellulose, hemicellulose and lignin contents}

The contents of cellulose, hemicellulose and lignin in IDF with bound phenolics and its alkaline hydrolysis residue (IDF without bound phenolics) were determined as described by Claye et al. (1996).

\subsection{Environmental Scanning Electron Microscopy (ESEM)}

The morphology and microstructural features of IDF with bound phenolics and its alkaline hydrolysis residue (IDF without bound phenolics) elucidated via an ESEM (Quanta 250 FEG, FEI Company, USA). The samples were sliced into rectangular shape in horizontal plane. The samples were then sputter coated with gold. The microstructural evaluation was performed at an operational voltage of $5 \mathrm{kV}$.

\section{$2.11 \mathrm{X}$-ray diffraction $(\mathrm{XRD})$}

A diffractometer (PANalytical X'Pert PRO, Almelo, Netherlands) was used to collect the XRD profiles of IDF with bound phenolics and its alkaline hydrolysis residue (IDF without bound phenolics). The copper Ka radiation $(0.154 \mathrm{~nm}, 40 \mathrm{kV}$, $50 \mathrm{~mA}$ ) from the instrument was set at a diffraction angle $(2 \theta)$ ranging from 4 to $40^{\circ}$.

\subsection{Fourier-transform infrared (FT-IR) spectroscopy}

The organic functional groups of IDF with bound phenolics and its alkaline hydrolysis residue (IDF without bound phenolics) were analyzed using a FT-IR spectrophotometer (FTIR/NIR 400, Perkin-Elmer Inc., Waltham, MA, USA). The spectrum wavelength was $400-4000 \mathrm{~cm}^{-1}$ at $4 \mathrm{~cm}^{-1}$ resolution with 4 scans at a scan speed of $<10 \mathrm{~s}$.

\subsection{Statistical analysis}

Triplicate fashion was adopted for all experimental measurements. The recorded data was presented as mean \pm standard deviation. One-way analysis of variance (ANOVA) was used to determine differences between treatments and were carried out in SPSS version 18.0 (Chicago, IL, USA). The differences in means were evaluated using the Tukey's test for means with $95 \%$ confidence limit $(p<0.05)$.

\section{Results and discussion}

\subsection{TPC and antioxidant activities}

TPC and antioxidant activities of SDF and IDF- bound phenolic complexes treated by alkaline hydrolysis in grape pomace were determined and results are tabulated in Table 1. IDF-bound phenolic complexes showed the highest TPC of $28.45 \mathrm{mg} / \mathrm{g}$ DW as compared to SDF fraction (12.09 mg/g DW) subjected to alkaline hydrolysis. In case of ABTS, the bound phenolic complexes of IDF fraction exhibited highly significant RSA value of $221.75 \mu \mathrm{mol}$ TE/100 g DW in comparison with that was determined for SDF treated by alkaline hydrolysis ( $9.89 \mu \mathrm{mol} \mathrm{TE} / 100 \mathrm{~g} \mathrm{DW})$. Similarly, IDF exhibited the highest FRAP $(306.86 \mu \mathrm{mol}$ TE/100 g DW) as compared to SDF $(26.71 \mu \mathrm{mol} \mathrm{TE} / 100 \mathrm{~g}$ DW). Bound phenolics are also known as non-extractable polyphenols (NEP's), and alkaline hydrolysis is one of the technical modality to achieve effective recovery of bound phenolics (Dzah et al., 2020). The possible reason for higher TPC of SDF-derived bound phenolics might the possibility of induction of cleavage due to alkaline $\mathrm{pH}$ between ester bonds existing between cell wall and phenolic acids which further led to achieve increased mass transfer of phenolic compounds from grape pomace matrix (Ding et al., 2020). Naturally, it is evident that change in bound phenolic content may exert significant effect on antioxidant capacity measured through ABTS and FRAP assays. The results of higher TPC, ABTS and FRAP-RSA of IDF-bound phenolics was in agreement with the findings reported by Cai et al. (2019) for phenolic profile and antioxidant capacity fermented Chinese rice wine.

\subsection{Identification of bound phenolic compounds of SDF and IDF treated by alkaline hydrolysis}

Bound phenolic profile compositions and respective contents of identified phenolic compounds were determined through HPLC and results are tabulated in Table 2. In both SDF and IDF alkaline extracts, total 7 and 6 phenolic compounds were detected through HPLC. In SDF alkaline extract, the identified phenolic compounds were as; gallic acid $(421.81 \mu \mathrm{g} / \mathrm{g})$, protocatechuic acid $(63.1 \mu \mathrm{g} / \mathrm{g})$, chlorogenic acid $(256.07 \mu \mathrm{g} / \mathrm{g})$, syringic acid $(45.41 \mu \mathrm{g} / \mathrm{g})$, epigallocatechin $(231.14 \mu \mathrm{g} / \mathrm{g})$, catechin $(451.07 \mu \mathrm{g} / \mathrm{g}), p$-coumaric acid $(62.21 \mu \mathrm{g} / \mathrm{g})$. Salicylic acid and ferulic acid showed undetected status in case of SDF alkaline extract. Conversely for IDF alkaline extract, the identified phenolic compounds were as; salicylic acid $(31.53 \mu \mathrm{g} / \mathrm{g})$, chlorogenic acid $(2494.53 \mu \mathrm{g} / \mathrm{g})$, syringic acid $(233.67 \mu \mathrm{g} / \mathrm{g})$, epigallocatechin $(956.1 \mu \mathrm{g} / \mathrm{g}), p$-coumaric acid $(706.14 \mu \mathrm{g} / \mathrm{g})$ and ferulic acid

Table 1. TPC, ABTS and FRAP of SDF and IDF treated by alkaline hydrolysis in grape.

\begin{tabular}{crc}
\hline & \multicolumn{1}{c}{ SDF } & \multicolumn{1}{c}{ IDF } \\
\hline TPC $(\mathrm{mg} / \mathrm{g} \mathrm{DW})$ & $12.09 \pm 0.06 \mathrm{~b}$ & $28.45 \pm 0.89 \mathrm{a}$ \\
ABTS $(\mu \mathrm{mol} \mathrm{TE} / 100 \mathrm{~g} \mathrm{DW})$ & $9.89 \pm 0.34 \mathrm{~b}$ & $221.75 \pm 24.87 \mathrm{a}$ \\
FRAP $(\mu \mathrm{mol} \mathrm{TE} / 100 \mathrm{~g} \mathrm{DW})$ & $26.71 \pm 0.26 \mathrm{~b}$ & $306.86 \pm 0.90 \mathrm{a}$ \\
\hline
\end{tabular}

Values represent means \pm standard deviations. Means followed by different letters (a-b) in the same row represent statistically significant differences $(p<0.05)$.

Table 2. Phenolic compounds of SDF and IDF treated by alkaline hydrolysis in grape.

\begin{tabular}{ccc}
\hline & SDF & IDF \\
\hline Gallic acid & $421.80 \pm 1.98$ & - \\
Protocatechuic acid, & $63.00 \pm 0.28$ & - \\
Salicylic acid & - & $31.53 \pm 5.35$ \\
Chlorogenic acid & $256.07 \pm 4.11 \mathrm{~b}$ & $2494.53 \pm 73.78 \mathrm{a}$ \\
Syringic acid & $45.40 \pm 0.20 \mathrm{~b}$ & $233.67 \pm 6.60 \mathrm{a}$ \\
Epigallocatechin & $231.13 \pm 11.79 \mathrm{~b}$ & $956.00 \pm 10.18 \mathrm{a}$ \\
Catechin & $451.07 \pm 6.52$ & - \\
p-Coumaric acid & $62.20 \pm 0.20 \mathrm{~b}$ & $706.13 \pm 29.71 \mathrm{a}$ \\
Ferulic acid & - & $60.00 \pm 3.11$ \\
\hline
\end{tabular}

Values represent means \pm standard deviations. Means followed by different letters (a-b) in the same row represent statistically significant differences $(p<0.05)$. 
$(60.01 \mu \mathrm{g} / \mathrm{g})$ whereas, gallic acid, protocatechuic acid and catechin were not detected in IDF alkaline extracts of grape pomace. It was clearly evident from the results that IDF-bound phenolics exhibited significantly $(p<0.05)$ higher quantities as compared to those were detected in SDF alkaline extract. Although, all detected compounds are of IDF-bound phenolics of grape pomace, however, each phenolic compound varied in terms of content and composition. These results were in agreement with the findings reported by Xu et al. (2020) for 11 identified bound phenolic compounds from IDF fraction of lychee pulp subjected to alkaline hydrolysis. It was also implied by the researchers that varietal difference may also exert its significant effect on compositions and contents of bound phenolics originated from plants (Xi et al., 2021). Previous studies have endorsed that the antioxidant potential of phenolic compounds depends on several factors, such as position and number of hydroxyl groups along with structural configuration of phenolics. Syringic acid and chlorogenic acid contain 1-2 and 5 hydroxyl groups, respectively. Actually chlorogenic acid is one of the predominantly existing class of bound phenolics in plants and chlorgenic acid actually forms as a result of esterification of caffeic acid and (-)-quinic acid, and quinic acid has been reported to possess four distinct hydroxyl groups in its structural configuration. These hydroxyl groups might also contribute to the total antioxidant capacity (TAC) of SDF and IDF extracts of grape pomace obtained by alkaline hydrolysis (Meng et al., 2019). Moreover, IDF-bound phenolics showed decreasing tendency with corresponding decreases in IDF. Hydroxybenzoic acids derivatives, such as protocatechuic acid were the bound components which were covalently bonded to the complex plant cell structures like lignin, whereas hydroxyl cinnamic acid derivatives, like $p$-coumaric acid, ferulic acid and chlorogenic acid are mainly present in bound form by means of ester linkages with cell wall structural components (lignin, cellulose and protein) (Dutra et al., 2018; Meng et al., 2019). The increased levels of bound phenolics in IDF extract in comparison with those observed in SDF fraction might be explicable in terms of release of polyphenols embedded with strong linkages in macromolecules of grape pomace after subjecting to alkaline hydrolysis (Sanz-Pintos et al., 2017). Exposure to alkaline hydrolysis has been reported to result in generation of several peroxide $\left(\mathrm{H}_{2} \mathrm{O}_{2}\right)$ reactive species and one of prominently produced is perhydroxyl anion (-). Under alkaline conditions, $\mathrm{H}_{2} \mathrm{O}_{2}$ may react with hydroperoxide anion, thus cause formation of superoxide anion radicals $\left(\mathrm{O}^{2-}\right.$. $)$ and hydroxyl radicals (HO). All these generated reactive moieties might lead to depolymerization effect on plant cell matrix, such as cellulose, lignin and hemicellulose (Vitaglione et al., 2008). Radicals might predominantly attack lignin as the preferred site of attack and hence, protocatechuic acid could also be attacked by radicals as hydroxybenzoic acid derivative. However, the possibility of alkaline hydrolysis treatment on degradation of original polyphenolic structures cannot be ruled out (Zheng \& $\mathrm{Li}, 2018)$. Alkaline hydrolysis may disrupt the three-dimensional structure of lignin in plant cell matrix and alteration of lignin structure might cause reduction of bound phenolics after exposure to alkaline treatment.

\subsection{Cellulose, hemicellulose and lignin of IDF with and without bound phenolics}

Results of cellulose, hemicellulose and lignin of IDF with bound phenolics and its alkaline hydrolysis residue (IDF without bound phenolics) in grape pomace are given in Table 3. It was evident from the results that hemicellulose and lignin were significantly $(p<0.05)$ lower in case of alkaline hydrolysis residue (IDF without bound phenolics) as compared to IDF fraction with bound phenolics. IDF with bound phenolics fraction exhibited the cellulose content of $22.31 \mathrm{mg} / \mathrm{g}$, whereas alkaline hydrolysis residue (IDF fraction without bound phenolics) had cellulose contents of $29.19 \mathrm{mg} / \mathrm{g}$. Both IDF fractions with and without bound phenolics exhibited hemicellulose contents of 20.15 and $15.24 \mathrm{mg} / \mathrm{g}$, respectively. Lignin contents for both fractions were also determined and were as; IDF fraction with bound phenolics ( $41.24 \mathrm{mg} / \mathrm{g}$ ) and IDF without bound phenolics $(30.36 \mathrm{mg} / \mathrm{g})$. Cellulose exhibited increasing trend with corresponding decreases in hemicelluloses and lignin contents. The probably reason or increases in cellulose content might be attributable to the decreases in non-fiber components or other small molecular-weighed polysaccharides. Decreases in lignin and hemicellulose content in alkaline residue fraction might be attributable to the alkalinity-induced degradation of hemicellulose and lignin leading to formation of perforations in DF whereas conversely, the exposure to alkaline hydrolysis treatment led to increased cellulose content in alkaline hydrolysis residue (IDF without bound phenolics) (Cai et al., 2021). Cell wall matrix majorly composed of cellulosic and hemi-cellulosic fractions whereas lignin is also found in embedded form throughout the cell-wall matrix with strong concentrations in regions of middle lamella. Cellulose is found in compositions of matrix consisted by combinations of pectin, proteins, and hemicelluloses (Zhang et al., 2020).

Hydrogen bonds are one of main forms of linkages which are responsible for bonding of both lignin and microfibrils. $\mathrm{H}_{2} \mathrm{O}_{2}$ acts as a strong oxidizing agent which causes degradation of lignin, hence it might be implied that $\mathrm{H}_{2} \mathrm{O}_{2}$ exposure led to possible decreasing tendencies in lignin and hemicellulose owing to $\mathrm{H}_{2} \mathrm{O}_{2}$-induced oxidizing effect (Gabriel et al., 2020). Peroxide becomes unstable under alkaline conditions and leads to formation of hydroxyl and superoxide radicals. Similar alkalinity-induced declining tendencies in hemicellulose and lignin have been reported by other researchers (Cai et al., 2021; Zhang et al., 2020).

Table 3. Cellulose, hemicellulose and lignin of IDF with bound phenolics and its alkaline hydrolysis residue (IDF without bound phenolics) in grape.

\begin{tabular}{ccc}
\hline & $\begin{array}{c}\text { IDF with bound } \\
\text { phenolics }\end{array}$ & $\begin{array}{c}\text { IDF without bound } \\
\text { phenolics }\end{array}$ \\
\hline Cellulose & $22.31 \pm 3.45 \mathrm{~b}$ & $29.19 \pm 1.45 \mathrm{a}$ \\
Hemicellulose & $20.15 \pm 1.81 \mathrm{a}$ & $15.24 \pm 1.32 \mathrm{~b}$ \\
Lignin & $41.24 \pm 3.29 \mathrm{a}$ & $30.36 \pm 2.61 \mathrm{~b}$ \\
\hline
\end{tabular}

Values represent means \pm standard deviations. Means followed by different letters ( $a-b)$ in the same row represent statistically significant differences $(p<0.05)$. 


\subsection{Microstructure of IDF with bound phenolics ( $A$ and $A_{1}$ ) and its alkaline hydrolysis residue (IDF without bound phenolics)}

Microstructure features of grape pomace IDF with bound phenolics and its alkali-hydrolyzed residue (IDF without bound phenolics) were determined by SEM at resolutions of 1500x and 3000x, respectively. The SEM micrographs of IDF with bound phenolics showed smooth surfaces at both 1500x and 3000x magnifications (Figures 1A and A1). IDF with bound phenolics of grape pomace exhibited curly flakes as were shown in SEM micrographs. Complex matrix of lignucellulosic biomass resulted in swelling of biomass substrate and caused structural expansion. SEM micrographs of alkali-hydrolyzed IDF revealed structural variations because of alkaline hydrolysis.

IDF with bound phenolics exhibited denser, more compact and tubular shape whereas, the surface features of alkali-hydrolyzed residue (IDF without bound phenolics) exhibited clear fragmentation, wrinkling and porosity on outside surfaces. Moreover, loose inner structures were evident in alkali-hydrolyzed residue (IDF without bound phenolics) of grape pomace. The loose inner structures and fragmentation of alkali-hydrolyzed residue were indicative of hydrolysis-induced breakage of grape pomace DF glycosidic linkages. The structural configuration of IDF did not show any significant damage after exposure to alkaline hydrolysis. Open, porous and loose structural attributes of alkali-hydrolyzed residue

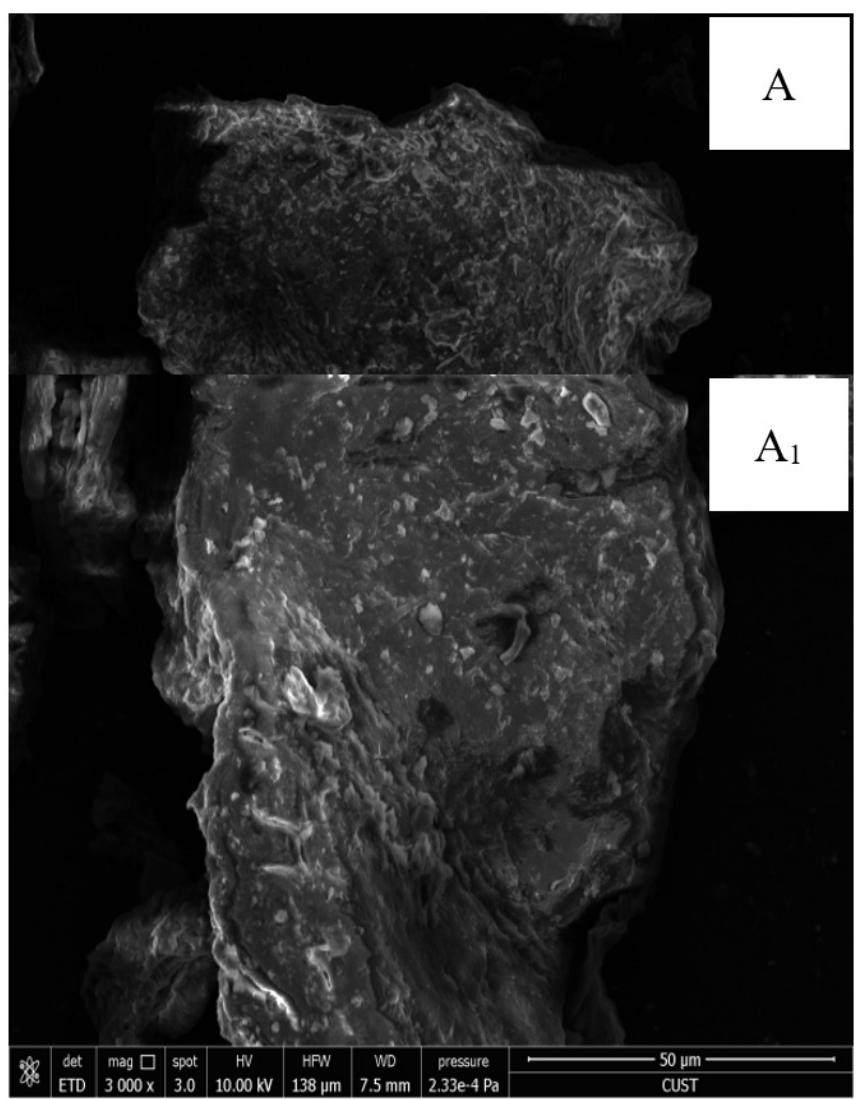

(IDF without bound phenolics) implied that strong alkaline conditions caused damage to IDF microstructure and led to formation of perforations and pores along with degradation of pectin, cellulose and hemicellulose fractions. It may have been also implied that strong alkaline conditions caused hydrolysis of many active substances existing in grape pomace DF in bound form and apparently led to partial fragmentation of dietary fibers. Similar results were reported in case of insoluble dietary fibers-bound phenolic complexes derived from lychee pulp and buckwheat straw insoluble dietary fibers by alkaline hydrolysis treatment (Meng et al., 2019; Xu et al., 2020).

\subsection{FT-IR spectroscopy of molecular structure characteristics of IDF samples and their alkaline hydrolysis residues}

FT-IR spectroscopy for elucidating molecular structure characteristics of IDF samples (with phenolics) and their alkali-hydrolyzed residue (IDF without bound phenolics) was carried out and results are presented in Figure 2. As evident from the FTIR spectra shown in Figure 2, both IDF samples (with phenolic) and alkali-soluble residue (IDF without phenolics) exhibited resemblance in FTIR spectral features and characteristics bonds. For instance, FTIR spectra at spectral regions of $3500-3200 \mathrm{~cm}^{-1}$ and $3500-3200 \mathrm{~cm}^{-1}$ had demonstrated two broad characteristic peaks which were indicative of stretching vibrations of $\mathrm{C}-\mathrm{H}$, and $\mathrm{O}-\mathrm{H}$ of carbohydrates and fiber and non-fiber components. The spectral peak at $2926 \mathrm{~cm}^{-1}$ was evident of presence of

Figure 1. Microstructure of IDF with bound phenolics ( $A$ and $A_{1}$ ) and its alkaline hydrolysis residue (IDF without bound phenolics) $\left(B\right.$ and $B_{1}$ ) in grape. A (x1500); $\mathrm{A}_{1}(\mathrm{x} 3000) ; \mathrm{B}(\mathrm{x} 1500) ; \mathrm{B}_{1}(\mathrm{x} 3000)$. 


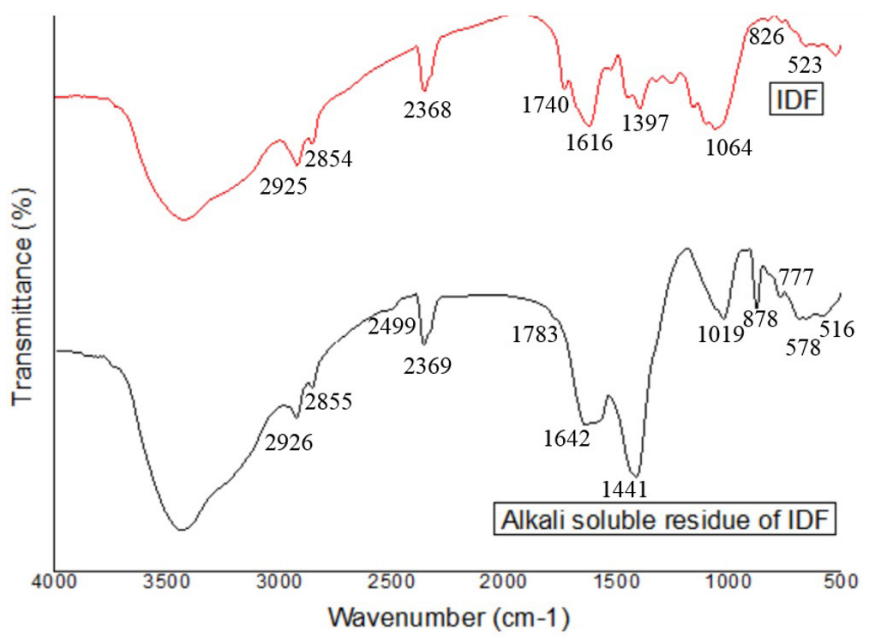

Figure 2. FTIR spectra showing wavenumbers for specific functional groups in IDF and Alkali residue IDF.

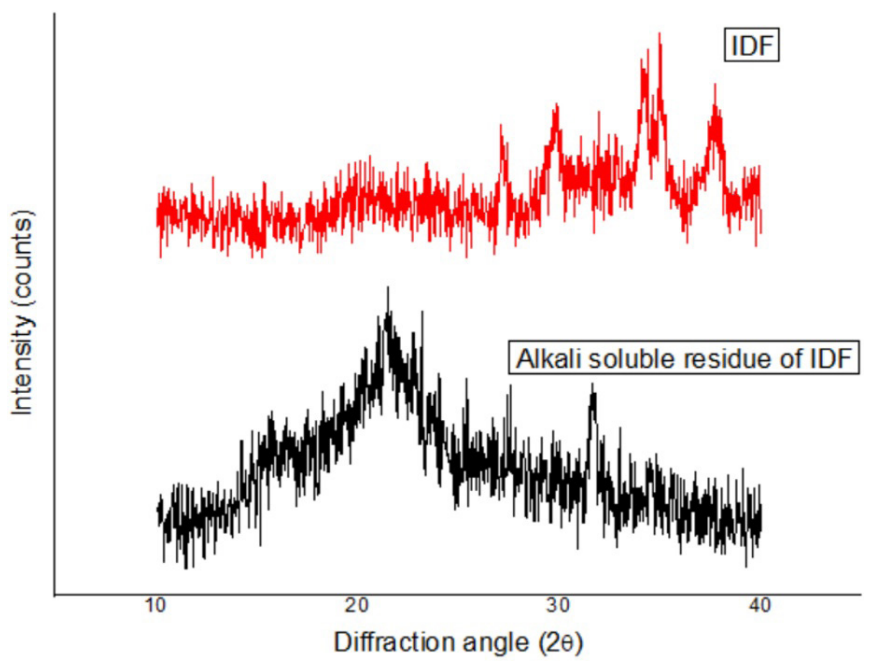

Figure 3. XRD diffractograms showing crystalline structural characteristics of IDF samples and alkali-hydrolyzed residues.

obvious linkages of intermolecular hydrogen bonding in IDF samples and alkali-hydrolyzed grape pomace residues (without bound phenolics). Strong absorption peaks in spectral ranges of 3000-2800 and $1400-1200 \mathrm{~cm}^{-1}$ were indicative of stretching vibration and characteristic angular vibration of carbohydrates. FTIR spectral peak at absorption region of $1642 \mathrm{~cm}^{-1}$ indicated presence of certain amount of crystalline water in IDF. Moreover, obvious peak at spectral region of $1441 \mathrm{~cm}^{-1}$ was indicative of $\mathrm{C}-\mathrm{O}$ vibrational stretching of carboxyl $(-\mathrm{COOH})$ functional group. After subjecting to alkaline hydrolysis treatment, the alkali-hydrolyzed residue did not show the characteristic peaks in infrared spectral regions of $1397 \mathrm{~cm}^{-1}$ and $2499 \mathrm{~cm}^{-1}$ in IDF without phenolics. This implied that grape pomace IDF phenolics were released from alkali-hydrolyzed residues after alkaline treatment. Overall, conclusively FTIR results showed that removal of bound phenolics from through alkaline hydrolysis did not cause any significant transitions of grape pomace IDF functional groups. These results were in agreement with findings of alkaline hydrolysis treatment for removal of bound phenolics from carrot dietary fiber (Liu et al., 2019b) and lychee pulp (Xu et al., 2020).

\subsection{XRD analysis for crystalline structural characteristics of IDF samples and alkali-hydrolyzed residues}

Crystalline structural properties of IDF samples and alkali-hydrolyzed residues were analyzed through XRD analysis and results are demonstrated in Figure 3 in terms of diffractograms. Both grape pomace IDF samples with phenolics and alkali-hydrolyzed residue (IDF without bound phenolics) exhibited similar prominent crystalline peaks at $21.67^{\circ}, 22.34^{\circ}$, indicated the typical cellulose 1 type diffraction angles whereas non-crystalline peaks were observed at $2 \theta$ angles of $25.38^{\circ}$ and $27.17^{\circ}$. Overall, the peak positions of IDF samples and alkali-hydrolyzed residue did not show any significant change, which was indicative of the fact that no major alterations were occurred in crystalline structures of IDF due to alkali-hydrolysis treatment (Meng et al., 2019). However, peak irregularities were observed in case of alkali-hydrolyzed residue at $2 \theta$ angles of $15.70^{\circ}, 21.67^{\circ}, 22.35^{\circ}, 25.38^{\circ}, 27.17^{\circ}, 32.71^{\circ}$, and $34.70^{\circ}$ when compared with diffractogram of IDF samples with phenolics.

Diffraction peaks irregularities and emergence of new peaks at aforementioned positions were possibly indicative of alkali hydrolysis-induced cellulose, hemicellulose and lignocellulosic fractions (Sheltami et al., 2012). Moreover, diffracted peaks exhibited higher crystalline intensity in alkali-soluble residue (without bound phenolics) as compared to IDF samples with bound phenolics, and the probable reason for this higher crystalline intensity might be attributable to the hydrolysis-induced disintegration of amorphous hemicellulose (Ma \& Mu, 2016). Moreover, it has already been reported by the previous researches that crystalline regions of IDF are primarily because of presence of cellulose, whereas non-crystalline components like non-crystalline cellulose, lignin and hemicellulose contributed to the overall amorphousness. Alkaline hydrolysis caused disintegration of linkages among hemicellulose and cellulose microfibers, lignin and hemicellulose in alkali-hydrolyzed residue and led to achieve increased mass transfer and solubilization in liquid fraction. Furthermore, the increased degree of crystallinity in alkali-hydrolyzed residue might resulted owing to removal of lignin and starch as well as hydrolysis of cellulosic amorphous regions and hemicelluloses (Wen et al., 2017). These results were in conformance with the microstructural changes elucidated by SEM micrographs (Figure 1). Similar results were reported for rice bran dietary fiber (Wen et al., 2017) and buckwheat straw insoluble dietary fiber by alkaline hydrogen peroxide treatment (Meng et al., 2019).

\section{Conclusions}

The novelty of this study was aimed at explanation of evolution process of bound phenolics from IDF through structural modifications after alkaline hydrolysis. ESEM results showed that IDF with bound phenolics exhibited denser, more compact and tubular shape whereas, the surface features of alkali-hydrolyzed residue (IDF without bound phenolics) 
exhibited clear fragmentation, wrinkling and porosity on outside surfaces. Microstructure changes due to alkaline hydrolysis caused disintegration of linkages among hemicellulose and cellulose microfibers. After subjecting to alkaline hydrolysis treatment, the alkali-hydrolyzed residue did not show the characteristic peaks in infrared spectral regions of $1397 \mathrm{~cm}^{-1}$ and $2499 \mathrm{~cm}^{-1}$ in IDF without phenolics. In both SDF and IDF alkaline extracts, total 9 phenolics compounds were detected through HPLC. The identified phenolic compounds were as; gallic acid, protocatechuic acid, salicylic acid, chlorogenic acid, syringic acid, epigallocatechin, catechin, $p$-coumaric acid and ferulic acid. The IDF content was higher than those in SDF of grape pomace. Dried grape pomace is a good source of IDF-with bound phenolics. Bound phenolics are also known as non-extractable polyphenols (NEP's), and alkaline hydrolysis is one of the technical modality to achieve effective recovery of bound phenolics.

\section{Conflict of interest}

The authors declare that they have no conflicts of interest.

\section{Funding}

This work was supported by Foundation of Ph.D. Research Project, Jilin Medical University (No. JYBS2019010), Jilin Province, China; Foundation of Science and Technology Department of Jilin Province (No. 20200404175YY), Foundation of the Education Department of Jilin Province (No. JJKH20170416KJ, jy2018xz04), Foundation of Health and Family Planning Commission of Jilin Province (No. 2019J066), Foundation of Suzhou Institute of medical engineering, Chinese Academy of Sciences-Jilin Science and technology cooperation project (No. E0550101), Jilin Collaborative Innovation Center for Antibody Engineering, Jilin Medical University (No. 20180623045TC).

\section{References}

Cai, H., Zhang, Q., Shen, L., Luo, J., Zhu, R., Mao, J., Zhao, M., \& Cai, C. (2019). Phenolic profile and antioxidant activity of Chinese rice wine fermented with different rice materials and starters. $L W T, 111$, 226-234. http://dx.doi.org/10.1016/j.lwt.2019.05.003.

Cai, Y., Huang, L., Chen, B., Su, J., Zhao, X., Zhao, M., Zhao, Q., \& van der Meeren, P. (2021). Effect of homogenization associated with alkaline treatment on the structural, physicochemical, and emulsifying properties of insoluble soybean fiber (ISF). Food Hydrocolloids, 113, 106516. http://dx.doi.org/10.1016/j.foodhyd.2020.106516.

Claye, S. S., Idouraine, A., \& Weber, C. W. (1996). In vitro mineral binding capacity of five fiber sources and their insoluble components for copper and zinc. Plant Foods for Human Nutrition, 49(4), 257-269. http://dx.doi.org/10.1007/BF01091975. PMid:8983052.

Ding, Y., Morozova, K., Scampicchio, M., \& Ferrentino, G. (2020). Nonextractable polyphenols from food by-products: current knowledge on recovery, characterisation, and potential applications. Processes, 8(8), 925. http://dx.doi.org/10.3390/pr8080925.

Drosou, C., Kyriakopoulou, K., Bimpilas, A., Tsimogiannis, D., \& Krokida, M. (2015). A comparative study on different extraction techniques to recover red grape pomace polyphenols from vinification byproducts. Industrial Crops and Products, 75, 141-149. http://dx.doi. org/10.1016/j.indcrop.2015.05.063.
Dutra, E. D., Santos, F. A., Alencar, B. R. A., Reis, A. L. S., Souza, R. F. R., Aquino, K. A. S., Morais, M. A. Jr., \& Menezes, R. S. C. (2018). Alkaline hydrogen peroxide pretreatment of lignocellulosic biomass: status and perspectives. Biomass Conversion and Biorefinery, 8(1), 225-234. http://dx.doi.org/10.1007/s13399-017-0277-3.

Dzah, C. S., Duan, Y., Zhang, H., Serwah Boateng, N. A., \& Ma, H. (2020). Latest developments in polyphenol recovery and purification from plant by-products: a review. Trends in Food Science \& Technology, 99, 375-388. http://dx.doi.org/10.1016/j.tifs.2020.03.003.

Eghdami, A., \& Sadeghi, F. (2010). Determination of total phenolic and flavonoids contents in methanolic and aqueous extract of Achillea millefolium. Organic Chemistry Journal, 2, 81-84.

Gabriel, T., Belete, A., Syrowatka, F., Neubert, R. H., \& Gebre-Mariam, T. (2020). Extraction and characterization of celluloses from various plant byproducts. International Journal of Biological Macromolecules, 158, 1248-1258. http://dx.doi.org/10.1016/j.ijbiomac.2020.04.264. PMid:32437811.

Guo, W., \& Beta, T. (2013). Phenolic acid composition and antioxidant potential of insoluble and soluble dietary fibre extracts derived from select whole-grain cereals. Food Research International, 51(2), 518525. http://dx.doi.org/10.1016/j.foodres.2013.01.008.

Irakli, M. N., Samanidou, V. F., Biliaderis, C. G., \& Papadoyannis, I. N. (2012). Development and validation of an HPLC-method for determination of free and bound phenolic acids in cereals after solid-phase extraction. Food Chemistry, 134(3), 1624-1632. http:// dx.doi.org/10.1016/j.foodchem.2012.03.046. PMid:25005991.

Jiang, G., Wu, Z., Ameer, K., Li, S., \& Ramachandraiah, K. (2020). Particle size of ginseng (Panax ginseng Meyer) insoluble dietary fiber and its effect on physicochemical properties and antioxidant activities. Applied Biological Chemistry, 63(1), 1-10. https://doi. org/10.1186/s13765-020-00558-2.

Li, Q., Yang, S., Li, Y., Huang, Y., \& Zhang, J. (2019). Antioxidant activity of free and hydrolyzed phenolic compounds in soluble and insoluble dietary fibres derived from hulless barley. $L W T, 111,534-540$. http:// dx.doi.org/10.1016/j.lwt.2019.05.086.

Liu, H. Y., Walden, T. B., Cai, D., Ahl, D., Bertilsson, S., Phillipson, M., Nyman, M., \& Holm, L. (2019a). Dietary fiber in bilberry ameliorates pre-obesity events in rats by regulating lipid depot, cecal short-chain fatty acid formation and microbiota composition. Nutrients, 11(6), 1350. http://dx.doi.org/10.3390/nu11061350. PMid:31208043.

Liu, S., Jia, M., Chen, J., Wan, H., Dong, R., Nie, S., Xie, M., \& Yu, Q. (2019b). Removal of bound polyphenols and its effect on antioxidant and prebiotics properties of carrot dietary fiber. Food Hydrocolloids, 93, 284-292. http://dx.doi.org/10.1016/j.foodhyd.2019.02.047.

Ma, M., \& Mu, T. (2016). Modification of deoiled cumin dietary fiber with laccase and cellulase under high hydrostatic pressure. Carbohydrate Polymers, 136, 87-94. http://dx.doi.org/10.1016/j. carbpol.2015.09.030. PMid:26572332.

Mañas, E., \& Saura-Calixto, F. (1993). Ethanolic precipitation: a source of error in dietary fiber determination. Food Chemistry, 47(4), 351355. http://dx.doi.org/10.1016/0308-8146(93)90176-G.

Maurer, L. H., Cazarin, C. B. B., Quatrin, A., Minuzzi, N. M., Costa, E. L., Morari, J., Velloso, L. A., Leal, R. F., Rodrigues, E., Bochi, V. C., Maróstica, M. R. Jr., \& Emanuelli, T. (2019). Grape peel powder promotes intestinal barrier homeostasis in acute TNBS-colitis: a major role for dietary fiber and fiber-bound polyphenols. Food Research International, 123, 425-439. http://dx.doi.org/10.1016/j. foodres.2019.04.068. PMid:31284994.

Meng, X., Liu, F., Xiao, Y., Cao, J., Wang, M., \& Duan, X. (2019). Alterations in physicochemical and functional properties of buckwheat straw 
insoluble dietary fiber by alkaline hydrogen peroxide treatment. Food Chemistry: X, 3, 100029. https://doi.org/10.1016/j.fochx.2019.100029.

Monteiro, G. C., Minatel, I. O., Pimentel, A. Jr., Gomez-Gomez, H. A., Camargo, J. P. C., Diamante, M. S., Pereira Basílio, L. S., Tecchio, M. A., \& Pereira Lima, G. P. (2021). Bioactive compounds and antioxidant capacity of grape pomace flours. LWT, 135, 110053. http://dx.doi.org/10.1016/j.lwt.2020.110053.

Sanz-Pintos, N., Pérez-Jiménez, J., Buschmann, A. H., Vergara-Salinas, J. R., Pérez-Correa, J. R., \& Saura-Calixto, F. (2017). Macromolecular antioxidants and dietary fiber in edible seaweeds. Journal of Food Science, 82(2), 289-295. http://dx.doi.org/10.1111/1750-3841.13592. PMid:28152188.

Sheltami, R. M., Abdullah, I., Ahmad, I., Dufresne, A., \& Kargarzadeh, H. (2012). Extraction of cellulose nanocrystals from mengkuang leaves (Pandanus tectorius). Carbohydrate Polymers, 88(2), 772-779. http://dx.doi.org/10.1016/j.carbpol.2012.01.062.

Thaipong, K., Boonprakob, U., Crosby, K., Cisneros-Zevallos, L., \& Byrne, D. H. (2006). Comparison of ABTS, DPPH, FRAP, and ORAC assays for estimating antioxidant activity from guava fruit extracts. Journal of Food Composition and Analysis, 19(6-7), 669-675. http:// dx.doi.org/10.1016/j.jfca.2006.01.003.

Vitaglione, P., Napolitano, A., \& Fogliano, V. (2008). Cereal dietary fibre: a natural functional ingredient to deliver phenolic compounds into the gut. Trends in Food Science \& Technology, 19(9), 451-463. http://dx.doi.org/10.1016/j.tifs.2008.02.005.
Wen, Y., Niu, M., Zhang, B., Zhao, S., \& Xiong, S. (2017). Structural characteristics and functional properties of rice bran dietary fiber modified by enzymatic and enzyme-micronization treatments. LWT, 75, 344-351. http://dx.doi.org/10.1016/j.lwt.2016.09.012.

Xi, Z., Gao, K., Guo, X., Li, M., \& Ren, C. (2021). Mechanistic study of the inhibition of active radicals in coal by catechin. Combustion Science and Technology, 193(11), 1931-1948. http://dx.doi.org/10. 1080/00102202.2020.1718122.

Xu, Z., Xiong, X., Zeng, Q., He, S., Yuan, Y., Wang, Y., Wang, Y., Yang, X., $\& \mathrm{Su}, \mathrm{D} .(2020)$. Alterations in structural and functional properties of insoluble dietary fibers-bound phenolic complexes derived from lychee pulp by alkaline hydrolysis treatment. LWT, 127, 109335. http://dx.doi.org/10.1016/j.lwt.2020.109335.

Zhang, Y., Qi, J., Zeng, W., Huang, Y., \& Yang, X. (2020). Properties of dietary fiber from citrus obtained through alkaline hydrogen peroxide treatment and homogenization treatment. Food Chemistry, 311, 125873. http://dx.doi.org/10.1016/j.foodchem.2019.125873. PMid:31780221.

Zheng, Y., \& Li, Y. (2018). Physicochemical and functional properties of coconut (Cocos nucifera L.) cake dietary fibres: effects of cellulase hydrolysis, acid treatment and particle size distribution. Food Chemistry, 257, 135-142. http://dx.doi.org/10.1016/j.foodchem.2018.03.012. PMid:29622189. 